\title{
$\mathfrak{I} \mathfrak{n} \mathfrak{b} \mathfrak{a} \mathfrak{l} \mathfrak{t}$
}

\section{Erites \$eft.}

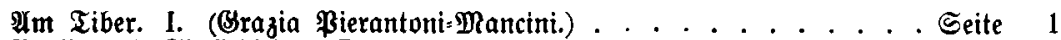

Boefie unb Sittlichfett. (Dtto நarnaf.). . . . . . . . . . . . . - 44

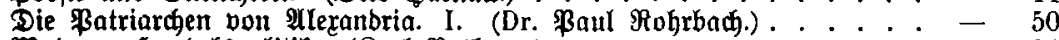

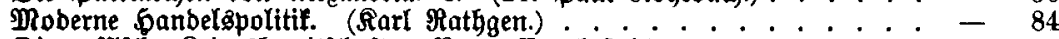

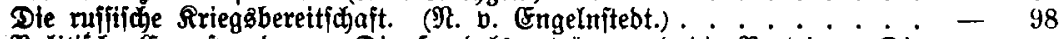

\$olitifije Sorrefponbenz: Die Sandeløberträge und bie \$arteien. Die

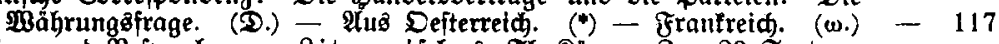

Notizen unb Befprechungen. \&iterarifめez: Th. Rörner, Bum 23 . Septem= ber 1891. - $\mathbb{X}$. Senfe, Borträge über $\mathfrak{B l a f t i f ,}$ Timif unb Drama. -

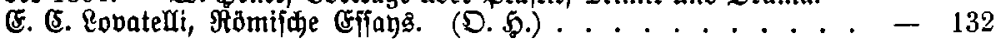

\section{3weites \$eft.}

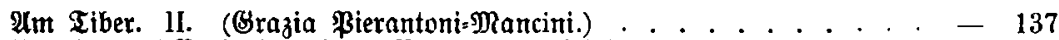

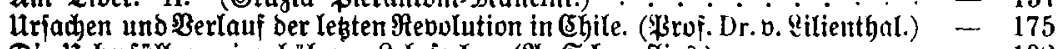

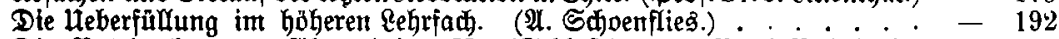

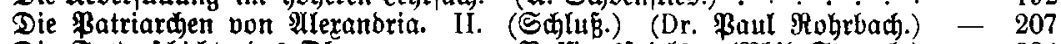

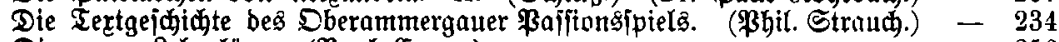

Die neuen Rehrpläte. (\$aul (Sauer.) . . . . . . . . - 256

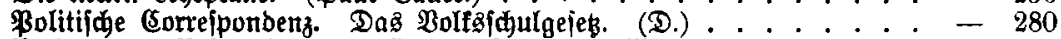

Notizen und Beipredungen: (Evnrab Fferbinand Mever, Angela Burgia. Rovelle. - Siegiried Szamatóläti, Das Fauf́tbud des (Ehriftid

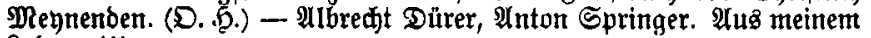
Regen. $(\dagger) \ldots \ldots . . . . . . . . . .290$

\section{Drittes \$eft.}

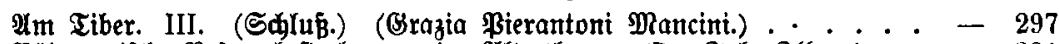

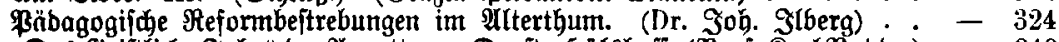

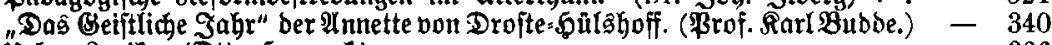

Ueber Inrif. (Dtto \$arnađ.). . . . . . . . . 386

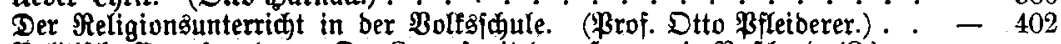

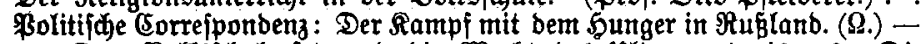

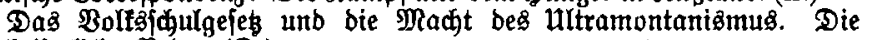

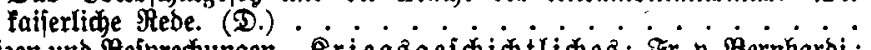

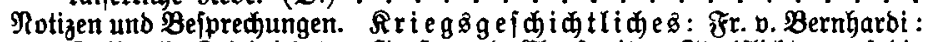

Delbrǘ, Frieorid Der Broge und Elaulemib. Streiflidter auf bie

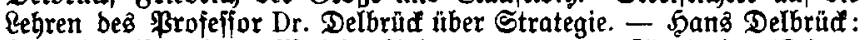
Friebrich, Mapoleon, Moltfe. Aleltere uno neuere Strategie. (D.) -

Mémoires du Général Bon de Marbot. (w.) . . . . . . . . - 434

\section{Biertes beft.}

3oroajter. I. (F. Marion (Erawforb.) . . . . . . . . . . . . 437

Heber bie Entroiđelung beş ßrobbetriebes und bie joztale Rlaffenbilbung.

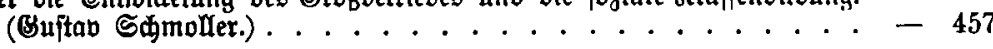




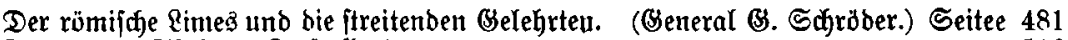

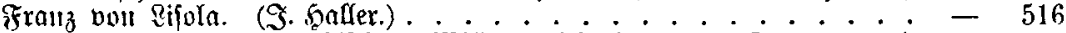

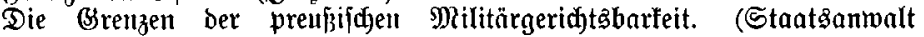
Itr. Damme.) . . . . . . . . . . . . . . .

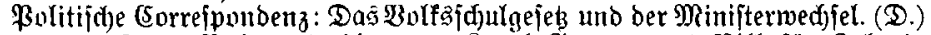
- Some $\mathfrak{R}$ ulle und bie nette Rocal \&opernment $\mathfrak{B}$ ill für Şrlant.

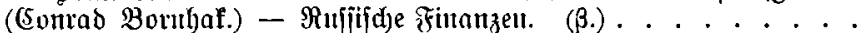

Plotizen umb Befpredfungen. Riterarifd es : Seinrid Freiferr Eangwerth vou Simmern, $\mathfrak{A}$ us ber $\mathfrak{M a p p e}$ eines beriturbenen Frembes (Friedrich

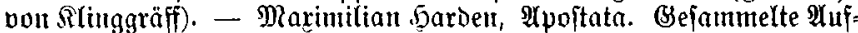

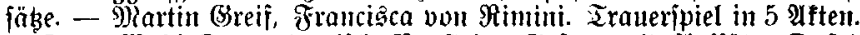

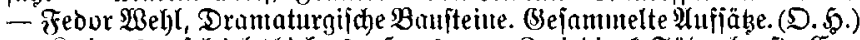

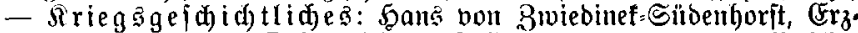
herzog Sobam von Defterreid im Felbzuge von 1809. - Moltfes

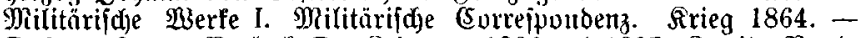
Dafor v. Qettuv= Borber, Der Arieg won 1806 unb 1807. Bweiter $\mathcal{B}$ anb. - Ridh. Sifhntitt, Die (Befechte bei Trautentau an 27. unt 28. Sonni 1866. (D.) - $\mathfrak{A} r$ d) äologie: Die Sartophage von હibon. (S.) -

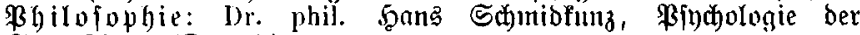
Eluggeptiont. (Drem. . . . . . . . . . . .

\section{Fünftes $\mathfrak{g e f t}$.}

Burvafter. II. (F. Marion (Eranford.) . . . . . . . . . . . . . - 593

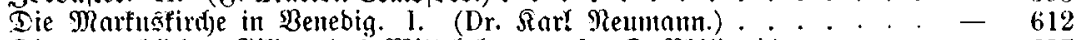

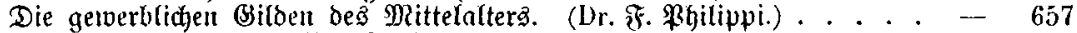

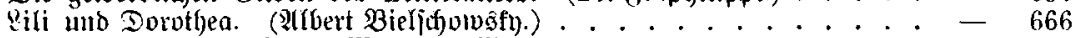

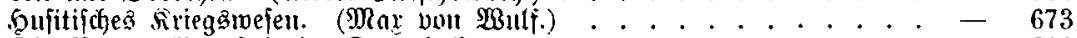

Tie Bergnerfinduitrie int Donezbeden . . . . . . . . . . . 690

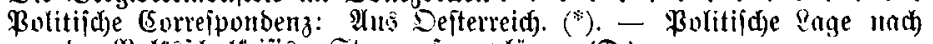

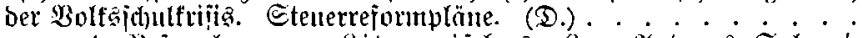

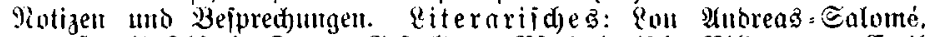

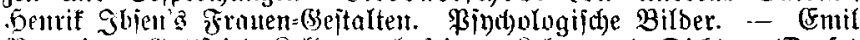

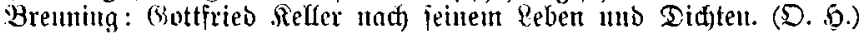

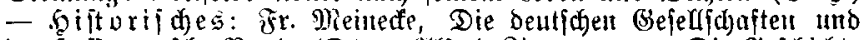

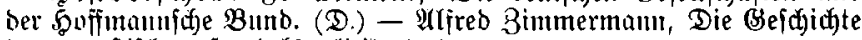

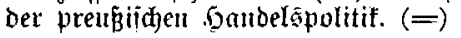

\section{Gedyftes \$eft.}

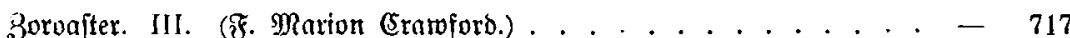

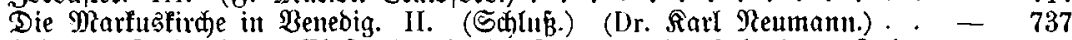

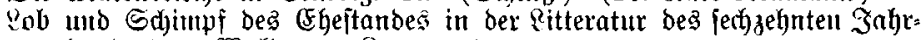

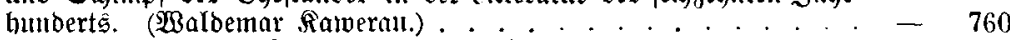

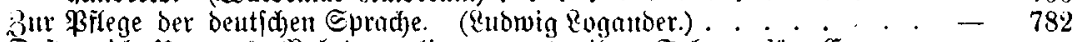

Depterreid) = Alugarns Balutaregulirung uno it)re Folgent jür (Europa. (Dr. Milliam Sitharling.)

Bolitithe Correipondenz: Die Berlenmoungen des bentichen Berwehrs. (D.)

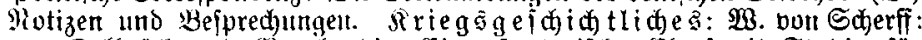

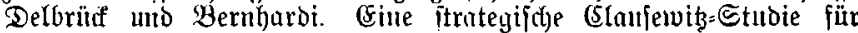
Bielehrte uno Milität $\alpha$. (Selbrït.) -

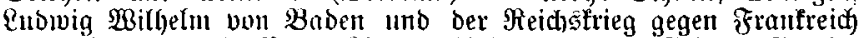

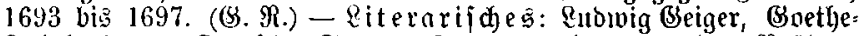
Sahrbud). - Dentfde Sagen. Serauggegeben pon ben Brübern Grimm. (‥5.) - Das Qeben Der Brinzeffin Elyarlotte Amelie be la Tremoille, Bräfin von \$llbenburg 1652-1732. Erzählt von thr

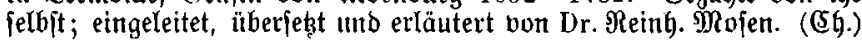

